\title{
Distribuição e abundância relativa de cumbaca Trachelyopterus striatulus Steindachner (Osteichthyes, Auchenipteridae) no reservatório de Lajes, Rio de Janeiro, Brasil
}

\author{
Silvana Duarte ${ }^{1}$ \\ Carla de Brito Caetano ${ }^{1}$ \\ Rafaela Nascimento Vicentini ${ }^{1}$ \\ Francisco Gerson Araújo ${ }^{1}$
}

\begin{abstract}
Distribution and relative abundance of the catfish Trachelyopterus striatulus Steindachner (Osteichthyes, Auchenipteridae) in the Lajes Reservoir, Rio de Janeiro, Brazil. The catfish Trachelyopterus striatulus Steindachner, 1877 is a very abundant fish species in reservoirs in Southeast Brazil, and its relative abundance seems to be increasing in several lentic environments. Despite of being a very common species, few information is available on its distribution and other aspects of ecology. The Lajes reservoir $\left(22^{\circ} 42^{\prime}-22^{\circ} 50^{\prime} \mathrm{S}\right.$ e $\left.43^{\circ} 53^{\prime}-44^{\circ} 05^{\prime} \mathrm{W}\right)$ is the largest manmade lake for hydropower purposes in Rio de Janeiro State, where this species rank among the top abundant fishes, both in number and in biomass. Its relative abundance were compared in three zones of the reservoir (upper, central and lower), based on a standardized program, during three annual periods (monthly sampling in 1994 and bi-monthly sampling in 1996 and 1997), with a total of three hundred twenty samples, using gill nets. Environmental variables of water temperature, $\mathrm{pH}$ and transparency were taken in each sampling occasion, and information on rainfall and level of the water were recorded. Trachelyopterus striatulus showed an increasing abundance during the study period, rising from fifty rank in 1994 to the most abundant fish in 1997, coinciding with decreasing water level, and suggesting that this species take advantage of the harsh conditions in the reservoir to increase population. Highest number and weight were recorded in the upper zone, which presented the lowest transparency. Seasonally no significance $(p>0,05)$ difference in abundance was found over the whole period.

KEY WORDS. Auchenipteridae, distribution, abundance, environmental parameters, Lajes, reservoir
\end{abstract}

Membros da família Auchenipteridae, conhecidos como cangatis ou cumbacas, encontram-se distribuídos em rios e represas de toda a América do Sul tropical estendendo-se desde o Panamá até a Argentina (NELSON 1976). Esta família é representada no reservatório de Lajes por uma única espécie, $T$. striatulus Steindachner, 1877, e pouco é conhecida sobre sua distribuição e abundância, apesar de suas elevada ocorrência.

1) Laboratório de Ecologia de Peixes, Instituto de Biologia, Universidade Federal Rural do Rio de Janeiro. Antiga Rodovia Rio -São Paulo Km 47, 23851-970 Seropédica, Rio de Janeiro, Brasil. E-mail: gerson@ufrrj.br

Revta bras. Zool. 19 (3): 925 - 933, 2002 
ARAúJO (1996) citou a ocorrência de T. striatulus (= Parauchneipterus striatulus) em baixas abundâncias relativas no rio Paraíba do Sul. Já no ambiente lêntico do reservatório de Lajes, que tem sua formação a partir do desvio de um tributário do rio Paraíba do Sul, esta espécie é uma das que apresenta maior abundância relativa (ARAÚJO \& SANTOS 2001). Um crescente aumento na abundância relativa destas espécies vem ocorrendo nos últimos anos no reservatório de Lajes (DUARTE \& ARAÚJO 2001). Este aumento em águas represadas ou mesmo em pequenas lagoas naturais tem sido recentemente comentado por pescadores profissionais e esportivos desta região do Brasil, onde a espécie é tida como indesejável pelo seu pequeno porte e por apresentar acúleos nas nadadeiras dorsais, que causam danos nas redes de pesca, além de pequenos acidentes na manipulação deste peixe. O presente trabalho tem por objetivo determinar os padrões de distribuição espacial e temporal desta espécie no reservatório de Lajes, e encontrar eventuais relações com os parâmetros ambientais que possam estar influenciando na ocorrência.

\section{MATERIAL E MÉTODOS}

O reservatório de Lajes ( $22^{\circ} 42^{\prime}$ - $22^{\circ} 50^{\prime}$ 'S; $\left.43^{\circ} 53^{\prime}-44^{\circ} 05^{\prime} \mathrm{W}\right)$ está localizado nas vertentes da Serra do Mar entre os municípios de Piraí e Rio Claro, a uma distância de aproximadamente $80 \mathrm{~km}$ da cidade do Rio de Janeiro, sendo o maior ambiente de águas represadas do Estado, e que foi construído com fins de produção de energia hidrelétrica no início do século passado. Em sua cota de $415 \mathrm{~m}$ acima do nível do mar, compreende cerca de $30 \mathrm{~km}^{2}$ de espelho líquido superficial (Fig. 1), com suas águas apresentando boa qualidade, sendo classificadas como oligomesotróficas, por apresentarem concentrações dos nutrientes nitrogênio e fósforo relativamente baixas, elevada transparência e baixas concentrações de pigmentos fotossintetizados por unidade de volume (BARROSO 1989; FEEMA 1991). O clima predominante é tropical úmido de altitude, com pluviosidade variável, sendo a média anual de $1700 \mathrm{~mm}$, temperatura média de $20^{\circ} \mathrm{C}$, com verões mais brandos e sem estação seca. O nível da água varia em função da pluviosidade e da demanda de água para usinas hidrelétricas, sendo mais elevado de março a junho, e mais baixo de setembro a dezembro (LIGHT/IESA 1991).

Foram realizadas 22 excursões ao reservatório entre janeiro de 1994 e dezembro de 1997. No período de janeiro de 1994 a dezembro de 1994 realizaram-se excursões mensais, e entre janeiro de 1996 a dezembro de 1997, excursões bimestrais; excepcionalmente, durante o ano de 1995 não foram efetuadas pescarias. Utilizou-se embarcação de $7 \mathrm{~m}$ de comprimento, impulsionado por motor de popa de $25 \mathrm{HP}$. Nas 320 amostragens, foram utilizadas redes de espera de $50 \mathrm{~m}$ de comprimento cada, $3 \mathrm{~m}$ de altura e malha variando de $2,5 \mathrm{~cm}$ e $6,5 \mathrm{~cm}$ de distância entre nós consecutivos. As redes foram colocadas ao entardecer de um dia e retiradas na manhã do dia seguinte. Juntamente com as coletas de peixes, foram medidos os fatores ambientais de temperatura, $\mathrm{pH}$ e transparência. Dados de pluviosidade e nível da água (cota) foram tomadas junto à estação meteorológica da Light Serviços de Eletricidade S/A, concessionária do reservatório de Lajes. 


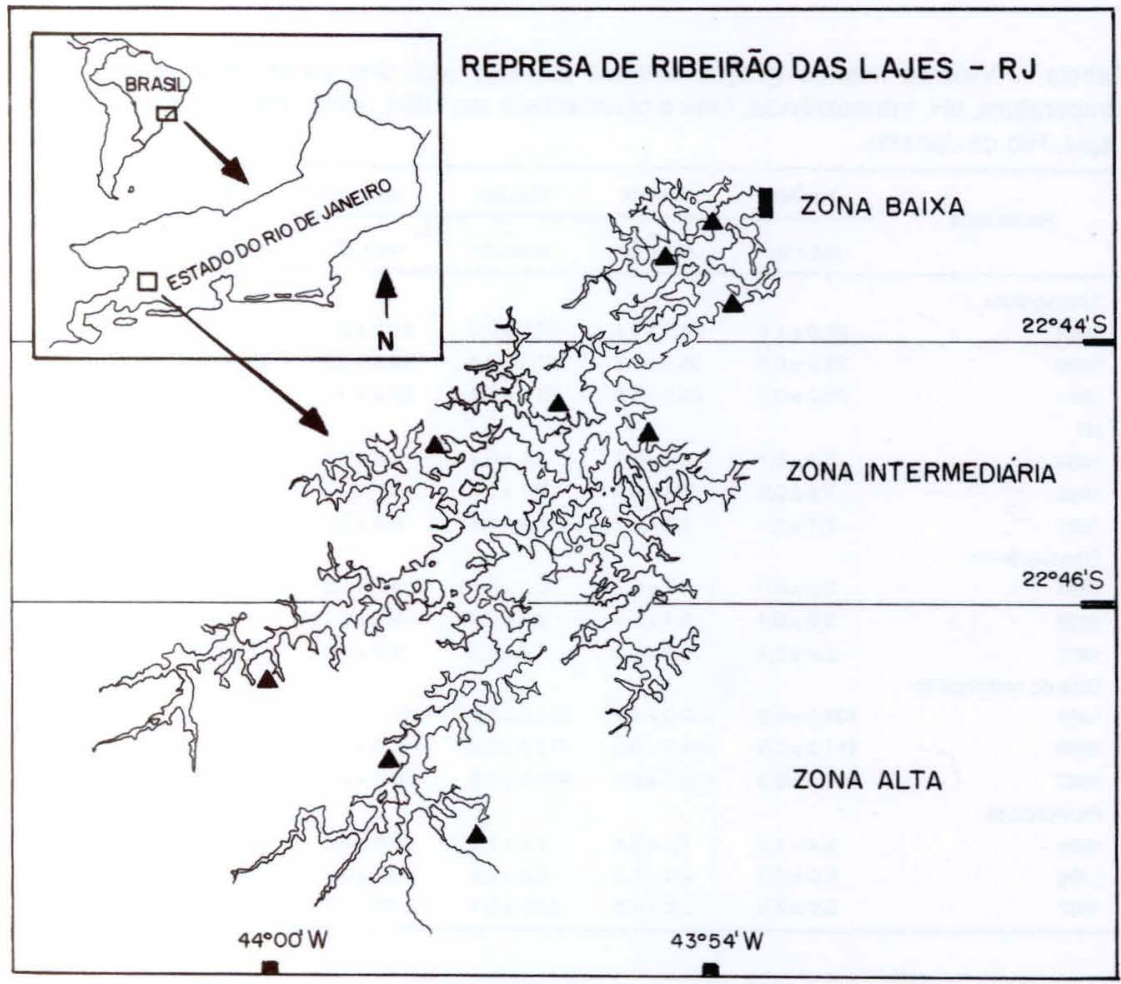

$\Delta$ - ÁREAS dE COLETAS

Fig. 1. Área de estudo, reservatório de Lajes, Rio de Janeiro, mostrando as três zonas de coletas.

Para efeito de comparações espaciais da abundância relativa da espécie e dos parâmetros ambientais, o reservatório foi dividido arbitrariamente em três zonas: 1) Zona Alta, próxima aos principais contribuintes lóticos de formação do reservatório; 2) Zona Intermediária e 3) Zona Baixa, próxima à barragem do reservatório. Em cada zona foram escolhidos aleatoriamente três locais de amostragem.

As comparações espaciais na abundância relativa e nos parâmetros ambientais foram feitas através de análise de variância não paramétrica de KruskalWallis (ZAR 1999); para os casos em que foram encontradas diferenças significantes, foi aplicado o teste não paramétrico de Mann-Withney (SOKAL \& RoHLF 1998). Também foram testadas correlações não paramétricas empregando o coeficiente $r$ de Spearman, entre a abundância dos peixes e os parâmetros ambientais examinados.

\section{RESULTADOS}

\section{Parâmetros ambientais (Tab. I)}

Temperatura. A temperatura variou sazonalmente com menores valores entre maio e setembro e maiores entre outubro e abril. Diferenças significativas 
Tabela I. Valores médios (md) e desvios padrões (dp) dos parâmetros ambientais de temperatura, $\mathrm{pH}$, transparência, cota e pluviosidade em 1994, 1996, 1997, no reservatório de Lajes, Rio de Janeiro.

\begin{tabular}{|c|c|c|c|c|c|c|}
\hline \multirow{2}{*}{ Parâmetros } & Jan/Fev & $\mathrm{Mar} / \mathrm{Abr}$ & Mai/Jun & Jul/Ago & Set/Out & Nov/Dez \\
\hline & $m d \pm d p$ & $m d \pm d p$ & $m d \pm d p$ & $m d \pm d p$ & $\mathrm{md} \pm \mathrm{dp}$ & $m d \pm d p$ \\
\hline \multicolumn{7}{|l|}{ Temperatura } \\
\hline 1994 & $26,0 \pm 1,6$ & $26,5 \pm 1,4$ & $23,0 \pm 0,4$ & $21,0 \pm 0,7$ & $25,0 \pm 1,9$ & $28,0 \pm 2,4$ \\
\hline 1996 & $29,0 \pm 0,9$ & $26,0 \pm 0,7$ & $23,5 \pm 0,8$ & $19,8 \pm 0,3$ & $23,9 \pm 0,6$ & $25,9 \pm 1,4$ \\
\hline 1997 & $28,2 \pm 0,2$ & $29,0 \pm 1,0$ & $22,3 \pm 0,4$ & $22,0 \pm 0,7$ & $25,0 \pm 1,5$ & $26,0 \pm 0,4$ \\
\hline \multicolumn{7}{|l|}{$\mathrm{pH}$} \\
\hline 1994 & $7,2 \pm 0,1$ & $8,0 \pm 0,3$ & $7,0 \pm 0,2$ & $7,0 \pm 0,1$ & $7,1 \pm 0,3$ & $7,8 \pm 0,4$ \\
\hline 1996 & $7,9 \pm 0,5$ & $7,9 \pm 0,5$ & $7,7 \pm 0,3$ & $8,1 \pm 0,4$ & $8,2 \pm 0,1$ & $7,9 \pm 0,3$ \\
\hline 1997 & $7,7 \pm 0,1$ & $7,6 \pm 0,2$ & $7,5 \pm 0,2$ & $6,9 \pm 0,2$ & $7,0 \pm 0,4$ & $7,9 \pm 0,3$ \\
\hline \multicolumn{7}{|l|}{ Transparência } \\
\hline 1994 & $2,5 \pm 0,0$ & $2,7 \pm 0,3$ & $2,4 \pm 0,5$ & $3,0 \pm 1,2$ & $2,8 \pm 0,7$ & $3,2 \pm 0,5$ \\
\hline 1996 & $3,0 \pm 0,4$ & $3,4 \pm 0,4$ & $3,5 \pm 0,6$ & $5,0 \pm 0,1$ & $4,0 \pm 0,0$ & $1,7 \pm 0,5$ \\
\hline 1997 & $2,9 \pm 0,4$ & $3,0 \pm 0,3$ & $2,2 \pm 0,6$ & $2,2 \pm 1,0$ & $2,4 \pm 0,2$ & $2,6 \pm 0,1$ \\
\hline \multicolumn{7}{|c|}{ Cota do reservatório } \\
\hline 1994 & $408,5 \pm 0,7$ & $410,0 \pm 1,7$ & $412,0 \pm 0,0$ & $411,7 \pm 0,4$ & $409,8 \pm 0,4$ & $409,0 \pm 0.0$ \\
\hline 1996 & $411,3 \pm 0,8$ & $414,0 \pm 0,0$ & $412,8 \pm 0,8$ & $410,5 \pm 0,5$ & $408,3 \pm 0,8$ & $406,5 \pm 0,1$ \\
\hline 1997 & $408,3 \pm 0,8$ & $410,5 \pm 0,5$ & $410,5 \pm 0,5$ & $406,3 \pm 2,8$ & $403,5 \pm 0,0$ & $404,0 \pm 0,5$ \\
\hline \multicolumn{7}{|l|}{ Pluviosidade } \\
\hline 1994 & $3,4 \pm 1,3$ & $7,1 \pm 2,8$ & $2,5 \pm 1,5$ & $0,5 \pm 0,4$ & $1,2 \pm 0,4$ & $4,6 \pm 1,8$ \\
\hline 1996 & $8,0 \pm 3,0$ & $5,0 \pm 1,8$ & $0,9 \pm 0,5$ & $0,5 \pm 0,3$ & $2,8 \pm 1,0$ & $63 \pm 2,4$ \\
\hline 1997 & $5,6 \pm 2,8$ & $5,0 \pm 1,8$ & $0,82 \pm 0,4$ & $0,23 \pm 0,1$ & $1,87 \pm 0,5$ & $4,45 \pm 1,8$ \\
\hline
\end{tabular}

foram encontradas entre os maiores valores de janeiro a março e os menores de junho a setembro. Não foram verificadas diferenças significativas entre as zonas nem entre os anos de estudo.

pH. Maiores valores de pH foram apresentados no ano de 1996 quando comparados com 1994 e 1997. Em relação aos meses, junho a agosto apresentaram menores valores em relação aos outros meses do ano, com os picos sendo alcançados em setembro e outubro. Maiores valores foram encontrados nas zonas baixa e intermediária quando comparados com a zona alta $(\mathrm{p}<0,05)$.

Transparência. Verificou-se significante diferença entre os anos, sendo que em 1996 a transparência foi relativamente maior do que o ano de 1994, e este, por sua vez, foi maior do que o ano de 1997. Os meses de junho a outubro apresentaram maiores transparências do que janeiro a maio. Espacialmente, não foram apresentadas diferenças significativas entre as zonas.

Cota. Variações significativas foram apresentadas durante os anos e bimestres, com interações entre estes dois fatores. Em geral, as maiores cotas foram apresentadas no ano de 1996, seguido de 1994, e menores em 1997, com picos entre janeiro e agosto, e menores valores de setembro a dezembro.

Pluviosidade. Foram encontradas significativas variações entre os bimestres, não tendo ocorrido variações entre os anos. O período entre novembro e abril foi caracterizado por apresentar maiores precipitações, enquanto de maio a outubro ocorreram as menores, com o mesmo padrão sendo consistente para os três anos. 


\section{Abundância relativa}

Número. Em 1994, esta espécie distribuiu-se de janeiro/abril em maior abundância nas zonas alta e intermediária, ocorrendo de maneira mais ampla em todas as três zonas de maio a outubro, e concentrando-se na zona baixa em novembro/dezembro, quando foram registradas as menores capturas, em oposição a março/abril, que apresentaram as maiores capturas (Fig. 2). Em 1996, o padrão de distribuição foi muito assemelhado ao de 1994, com maiores abundâncias em janeiro/abril, também se espalhando eqüitativamente nas três zonas de maio a outubro, e concentrando-se nas zonas alta e intermediária, em novembro/dezembro; as maiores abundâncias em geral ocorreram em março/abril e novembro/dezembro. Em 1997, as abundâncias foram relativamente mais elevadas em todos os meses de ano, com maiores registros em janeiro/abril na zona intermediária; de maio a outubro observou-se um padrão de maior abundância na zona baixa e menor, na zona alta, e em novembro/dezembro uma distribuição mais equilibrada entre as três zonas.

Em geral, verificou-se uma elevação gradativa na abundância numérica ao longo do período estudado, com menores ocorrências registradas em 1994, um relativo incremento ocorrendo em 1996 e maiores registros em 1997, quando foi a espécie de maior abundância relativa dentre todos os peixes da represa capturados em rede de espera. Não foram verificadas diferenças significativas na abundância entre os meses de cada um dos três anos. Espacialmente, a zona baixa apresentou menores valores em relação às zonas alta e intermediária (Tab. II)

Tabela II. Valores e significância de F da análise de variância para comparações entre os parâmetros ambientais e abundância de peixes (número e peso), no reservatório de Lajes, Rio de Janeiro. Diferenças significativas entre parênteses.

\begin{tabular}{|c|c|c|c|}
\hline Variáveis & Ano & Mês & Zona \\
\hline Temperatura & $\begin{array}{l}0,9 \\
\text { (ns) }\end{array}$ & $\begin{array}{c}171,6^{* *} \\
(j a n / m a r>j u n / \text { set })\end{array}$ & $\begin{array}{l}2,7 \\
\text { (ns) }\end{array}$ \\
\hline Transparência & $\begin{array}{c}78,9 * \\
(1996>1994>1997)\end{array}$ & $\begin{array}{c}35,1^{* *} \\
\text { (jun/out }>\text { jan/mai) }\end{array}$ & $\begin{array}{r}1,3 \\
\text { (ns) }\end{array}$ \\
\hline $\mathrm{pH}$ & $\begin{array}{c}53,4 \cdots \\
(1996>1994)\end{array}$ & $\begin{array}{c}57,8^{*} \\
\text { (set/out; } \text { mar > jun/ago) }\end{array}$ & $\begin{array}{c}6,9^{*} \\
(1=2>3)\end{array}$ \\
\hline Nivel da água (Cota) & $\begin{array}{c}92,5^{* *} \\
(1996>1994>1997)\end{array}$ & $\begin{array}{c}63,7^{* *} \\
(\mathrm{mar} / \mathrm{jun}>\mathrm{jan} / \mathrm{fev} ; \text { jul/ago > set/dez) }\end{array}$ & - \\
\hline Pluviosidade & $\begin{array}{l}0,5 \\
\text { (ns) }\end{array}$ & $\begin{array}{c}26,9^{* *} \\
(\text { jan/abr; nov/dez > mai/out) }\end{array}$ & - \\
\hline Número & $\begin{array}{c}5,6^{\circ} \\
(1997,1996>1994)\end{array}$ & $\begin{array}{l}11,1 \\
\text { (ns) }\end{array}$ & $\begin{array}{c}6,9^{\circ} \\
(2=3>1)\end{array}$ \\
\hline Peso & $\begin{array}{c}6,0^{\circ} \\
(1997,1996>1994)\end{array}$ & $\begin{array}{l}11,1 \\
\text { (ns) }\end{array}$ & $\begin{array}{c}6,0^{\circ} \\
(2=3>1)\end{array}$ \\
\hline
\end{tabular}

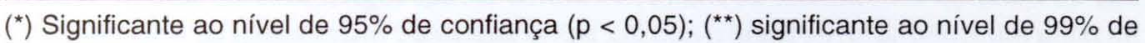
confiança $(p<0,01)$.

Peso. Em 1994, as maiores biomassas ocorreram em janeiro/fevereiro na zona intermediária; de março a maio, nas zonas alta e intermediária; de maio e outubro, com ampla e eqüitativa distribuição nas três zonas da represa; e em novembro/dezembro, nas zonas alta e intermediária (Fig. 3). Em 1996, as maiores 


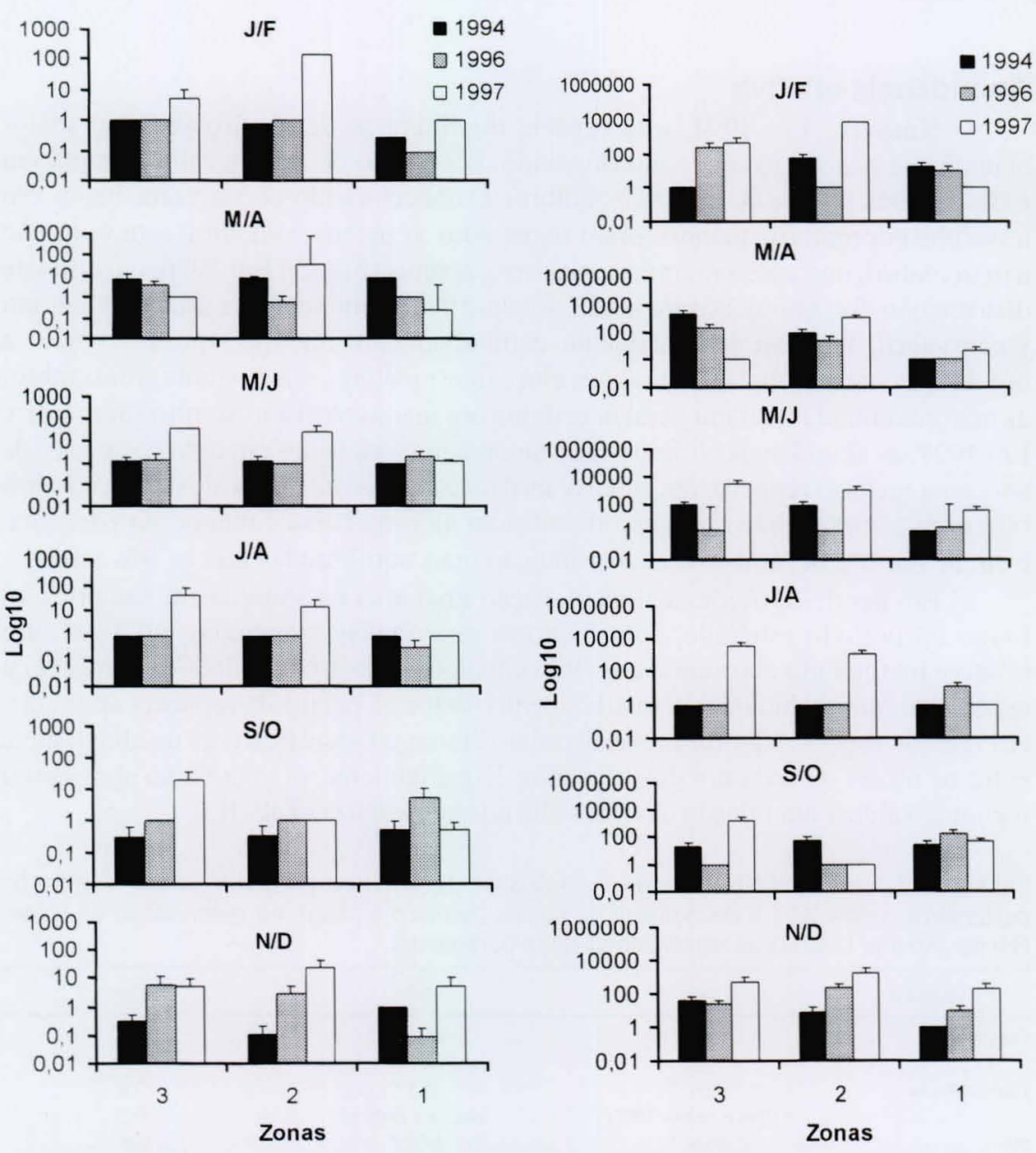

Figs 2-3. (2) Número de indivíduos $(\log 10)$ e (3) peso de individuos $(\log 10)$ por arrasto de $T$. striatulus no reservatório de Lajes, entre 1994 e 1997. Zonas de represas: (1) baixa, (2) intermediária, (3) alta.

abundâncias ocorreram de janeiro a abril na zona alta e intermediária; entre maio e agosto, de maneira eqüitativa nas três zonas; em setembro/outubro, na zona baixa; e em novembro/dezembro, na zona intermediária. Em 1997, quando foram registradas as maiores biomassas, os maiores valores foram registrados de janeiro a abril na zona intermediária; de maio a agosto, nas zonas alta e intermediária; setembro/outubro, na zona alta; e em novembro/dezembro, de maneira mais eqüitativa em todas as zonas. Em 1994 e 1996 as menores abundâncias ocorreram em julho/agosto, enquanto que em 1997, em setembro/dezembro.

O padrão de ocorrência em biomassa desta espécie foi muito similar ao da abundância numérica. Os maiores valores em biomassa ocorreram em 1997 e 1996, quando comparados a 1994. Espacialmente, a zona alta e a zona intermediária 
apresentaram maiores biomassas em relação à zona baixa. Não se verificaram diferenças significativas na abundância entre os bimestres de cada ano que pudesse sugerir sazonalidade (Tab. II).

\section{Influência dos parâmetros ambientais}

Dos parâmetros ambientais examinados, apenas a transparência apresentou associação significante $(\mathrm{p}<0,05)$ com os dados bióticos, tendo sido negativamente correlacionada com a abundância numérica e em biomassa, de acordo com o teste de correlação não paramétrica de Spearman (Tab. III).

Tabela III. Correlação não paramétrica ( $r$ - Sperman) significantes entre a abundância (numérica e biomassa) e os parâmetros ambientais.

\begin{tabular}{lccccc}
\hline \multirow{2}{*}{ Variáveis } & Temperatura & $\mathrm{pH}$ & Transparência & Cota & Pluviosidade \\
\hline Número & 0,07 & 0,01 & $-0,14^{*}$ & $-0,01$ & 0,07 \\
Peso & 0,07 & 0,01 & $-0,14^{*}$ & $-0,01$ & 0,07 \\
\hline
\end{tabular}

$\left({ }^{*}\right)$ Significante ao nível de $95 \%$ de confiança $(p<0,05)$.

\section{DISCUSSÃO}

Um dos aspectos mais relevantes na ocorrência de $T$. striatulus no reservatório de Lajes, foi a notável elevação da abundância relativa ao longo do período estudado, com aumento das densidades a partir do ano de 1994, início deste estudo, para o ano de 1997, quando esta espécie elevou suas contribuições do quinto para o primeiro lugar nas capturas de peixes com rede de espera, tanto em número como em peso (ARAÚJO \& SANTOS 2001). O ano de 1997 apresentou transparências e cotas (níveis de águas) mais baixas, quando comparados com os outros anos estudados, sugerindo que esta espécie tira proveito destas condições ambientais. Quando a cota é muito baixa, a maior parte da região litoral do reservatório encontra-se descoberta e a diversidade de habitat e disponibilidade de alimentos de origem alóctone é menor, uma vez que a vegetação marginal não se encontra encoberta, fatores estes que favorecem uma grande quantidade de peixes que dependem da elevação dos níveis da água para terem alimentação e proteção nos arbustos ou outro tipo de vegetação que sazonalmente é imersa.

No ano de 1997, quando os níveis de água foram mais baixos e a vegetação marginal não se encontrava encoberta, não era de se esperar que esta espécie apresentasse maiores abundâncias, uma vez que a disponibilidade de alimentos, especialmente os de origem alóctone, foi menor. Por outro lado, sua distribuição concentrada principalmente nas zonas alta e intermediária, onde existe maior entrada dos contribuintes (rios e riachos) que formam a represa, e que constituem fonte permanente de material alóctone, pode justificar a grande ocorrência desta espécie Peixes da família Auchenipteridae são citados por apresentarem uma dieta mista de vegetais e invertebrados, como microcrustáceos e insetos, principalmente de origem alóctone (FerReIRA 1984; MENEZES 1949).

O único parâmetro ambiental que apresentou significante correlação com a ocorrência de $T$. striatulus foi a transparência, com maiores abundâncias nos 
ambientes mais turvos. Os hábitos noturnos para a família Auchenipteridae tem sido reportado por SANDS (1984), que se refere citando a espécie $P$. galeatus Bleeker, 1863, bem como outros representantes da família, como Tatia Ribeiro, 1911, Trachycorystes Bleeker, 1862 e Centromochlus Kner, 1857. GARAVELLLO (1986) menciona estes mesmos hábitos para toda família Auchenipteridae, assim como LOWE-MCCONNEL (1975), ao referir-se à fauna de água doce da América do Sul. A grande ocorrência de $T$. striatulus, em menores transparências conforme observado neste trabalho, corrobora a preferência por ambientes com pouca luz, ou mesmo com o hábito noturno descrito pelos autores acima citados.

Uma possível explicação para a elevada abundância desta espécie poderia ser atribuída à estratégia reprodutiva. Trachycorystes galeatus Linneaus, 1756, uma espécie muito assemelhada ao T. striatulus, apresenta fecundação interna, como foi verificado por CHACON \& MENDES-FILHO (1972), o que indica a tendência à estratégia $\mathrm{k}$. Ao analisar as 10 espécies mais freqüentes na pesca experimental do reservatório de Itaipu durante os três primeiros anos de sua formação, AGOSTINHO (1994) registrou que três espécies de Auchenipteridae apresentavam também tendência à estratégia $\mathrm{k}$. Tal estratégia poderia estar sendo desenvolvida por $T$. striatulus no reservatório de Lajes, o que estaria contribuindo para as maiores abundâncias relativas independente das pressões ambientais, como no período de baixas cotas.

\section{REFERÊNCIAS BIBLIOGRÁFICAS}

Agostınho, A.A. 1994. Considerações acerca de pesquisas, monitoramento e manejo da fauna aquática em empreendimentos hidrelétricos. In: Seminário da Fauna Aquática do Setor Elétrico Brasileiro. Caderno, Rio de Janeiro, 1: 34-49.

ARaúso, F.G. 1996. Contribuição e estrutura da comunidade de peixes do médio e baixo Rio Paraíba do Sul - RJ. Rev. Brasil. Biol., Rio de Janeiro, 56 (1): 111-126.

ARaújo, F.G. \& L.N. Santos. 2001. Composition and structure of fish community in Laje's reservoir, RJ. Rev. Brasil. Biol., São Carlos, 61 (4): 563-576.

BARRoso, L. V. 1989. Diagnóstico ambiental para a pesca da águas interiores no Estado do Rio de Janeiro. Rio de Janeiro, IBAMA, Doc. ACUMEP 4, 117 .

Chacon, J. DE O. \& A. Mendes-Filho. 1972. Estudo morfológico do aparelho genital de Cangati, Trachycorystes galeatus Linneaus, 1756. Ci. Cult., São Paulo, 24 (6): 531-536.

DuARTE, S. \& F.G. ARAúJo. 2001. Abundância relativa e distribuição de Loricariichtys spixii (Steindachner) (Siluriformes, Loricariidae) no reservatório de Lajes, estado do Rio de Janeiro, Brasil. Revta bras. Zool, Curitiba, 18 (2): 465-477.

FEEMA (Fundação Estadual de Engenharia e Meio Ambiente). 1991. Qualidade das águas do Estado do Rio de Janeiro - período: 1989-1989. Relatório da Fundação Estadual de Engenharia e Meio Ambiente. Rio de Janeiro, Divisão de Qualidade de água, Departamento de Planejamento Ambiental, Vol. 2, 98p.

Ferreira, E.J.G. 1984. A ictiofauna da represa hidrelétrica de Curuá-Una, Santarém, Pará. II-Alimentação e hábitos alimentares das principais espécies. Amazoniana, Plön, 9 (1): 1-16.

Garavello, J.C. 1986. Fauna terrestre e aquática. In: Anais Simpósio sobre recursos naturais e sócio-econômicos do Pantanal, 1. Brasília, Departamento de Difusão e Tecnologia, p. 179-182.

LightлESA (Internacional de Engenharia S/A). 1991. Reservatório de Lajes- Estudo de viabilidade para o alceamento do nível d'água - Relatório Final SLP, 1-102, RE (RMD- 6102-RE). Rio de Janeiro, Vol. 1, 380p.

Lowe-MCCONNEL, R.H. 1975. Fish communities in tropical freshwaters: their distribution, ecology 
and evolution. London, Longman, 337p.

Menezes, R.S. DE. 1949. Alimentação de peixe gato, "Auchenipterus nuchalis" (Spix), da bacia do rio Parmaíba, Piauí (Actinopterygii, Doradidae, Auchenipteridae). Rev. Brasil. Biol., Rio de Janeiro, 9 (4): 489-495.

NELSON, J.S. 1976. Fishes of the world. New York, John Wiley \& Sons, 416p.

SANDS, D. 1984. Catfishes of the world. Dunure, Dunure Publications, Vol. 3, 49p.

SokAl, R.R. \& F.J. RohlF. 1998. Biometry. New York, W.H. Freeman, $3^{\text {rd }}$ ed., 887p.

Zar, J.H. 1999. Biostatistical Analysis. New Jersey, Prentice-Hall, $4^{\text {th }}$ ed., 663p.

Recebido em 14.XII.2001; aceito em 09.IX.2002. 